IP/BBSR/ 96-46

hep-th/9609025

\title{
A Quantum Many-body Problem in Two Dimensions: Ground State
}

\author{
Avinash Khare*and Koushik Ray ${ }^{\dagger}$ \\ Institute of Physics, \\ Bhubaneswar 751 005, INDIA
}

\begin{abstract}
We obtain the exact ground state for the Calogero-Sutherland problem in arbitrary dimensions. In the special case of two dimensions, we show that the problem is connected to the random matrix problem for complex matrices, provided the strength of the inverse-square interaction $g=2$. In the thermodynamic limit, we obtain the ground state energy and the pair-correlation function and show that in this case there is no long-range order.
\end{abstract}

Calogero-Sutherland type models are a class of exactly solvable models. Starting from the inception [1], till today, these models continue to be of interest, especially due to their exact solvability [2]. Moreover, these models are related to $(1+1)$-dimensional conformal field theory, random matrices and a host of other things [3]. Some variants of the models have attracted attention in very recent past [3 5]. The original model of Calogero [1] described $N$ particles in one dimension, interacting through an inverse square law and a two-body harmonic interaction. The latter interaction was added to the effect of complete discretization of the spectrum. This model gave in to an analytic treatment yielding an exact solution. Sutherland [6] considered a variant of this problem where the harmonic interaction was replaced by a harmonic well containing the $N$ particles. It was shown that one can exactly solve for the ground state of the system. The norm of the ground state wave-function was shown to be of a form that coincides with the joint probability density function of the eigenvalues of random matrices from an orthogonal, unitary or symplectic Gaussian ensemble, provided, the

\footnotetext{
*khare@iopb.ernet.in

$\dagger$ † koushik@iopb.ernet.in
} 
parameter giving the strength of the inverse-square interaction was fixed to $\frac{1}{2}, 1$ or 2 respectively. In an effort to generalize the original Calogero model [1] to dimensions higher than one, it was shown that some exact eigenstates including the ground state can be obtained for a three dimensional $N$-body problem with inverse-square interaction, provided one also adds a long-range three-body interaction [7], which is not present in one dimension. It is clearly of interest to enquire if this result of [7] could be generalized to arbitrary dimensions. Perhaps a much more interesting question is whether one could obtain the exact ground state for the Sutherland variant of this model in arbitrary dimensions. One could then hope that a la one dimensional case, one could map the problem to some random matrix problem and obtain exact results for the corresponding many-body problem. The purpose of this letter is to provide one such example. In particular, for the special case of two dimensions, we show that the norm of the ground state wave-function is related to the joint probability density function of the eigenvalues of complex random matrices, provided, the strength of the inverse square interaction $(g)$ is set to 2 . Using this correspondence, we obtain some exact results for the ground state of a two-dimensional many-body system in the thermodynamic limit. In particular, in the limit $N \longrightarrow \infty, \omega \longrightarrow 0$ with $N \omega$ fixed, we obtain the $n$-point correlation function. Explicit expressions for one- and two-point correlation functions are also given and it is pointed out that in this case $(g=2)$, unlike in one dimension, the particle density does not have the semi-circular form and the system does not have any long-range order.

Let us start by describing the model in $D$ dimensions. Following [7], we shall first consider the $N$-body problem in $D$ dimensions, defined by the Hamiltonian describing $N$ particles interacting through an inverse square potential, a harmonic potential and a three body term:

$$
\mathcal{H}=-\sum_{i=1}^{N} \nabla_{i}^{2}+g \sum_{\{i, j \mid i<j\}}^{N} \frac{1}{r_{i j}^{2}}+G \sum_{\substack{\{i, j, k\} \\ i<j, i \neq k, j \neq k}} \frac{\mathbf{r}_{k i} \cdot \mathbf{r}_{k j}}{r_{k i}^{2} r_{k j}^{2}}+\frac{1}{8} \omega^{2} \sum_{i=1}^{N} r_{i j}^{2},
$$

where we have chosen units such that $\hbar^{2}=2 m=1$. Following [7], it is easily shown that some eigenstates including the ground state for the system are given by,

$$
\Psi_{n}=\left(\prod_{i<j} r_{i j}^{2}\right)^{\frac{\Lambda_{D}}{2}} \exp \left(-\frac{\omega}{4} \sqrt{\frac{1}{2 N}} \sum_{\{i, j \mid i<j\}} r_{i j}^{2}\right) L_{n}^{\Gamma_{D}}\left(\frac{\omega}{2} \sqrt{\frac{1}{2 N}} \sum_{\{i, j \mid i<j\}} r_{i j}^{2}\right)
$$

with energy

$$
E_{n}=\sqrt{\frac{N}{2}}\left(2 n+\Gamma_{D}+1\right) \omega .
$$

Here $\mathbf{r}_{i}$ is the $D$-dimensional position vector of the $i$-th particle and $\mathbf{r}_{i j}=\mathbf{r}_{i}-\mathbf{r}_{j}$ denotes the relative separation of the $i$-th and $j$-th particles, while $r_{i j}$ denotes its magnitude. The parameters $g$ and $G$ are the strengths of the two- and three-body 
potentials, respectively. Here $g>-1 / 2$ to stop the fall to the origin. Further, $\Lambda_{D}$ and $\Gamma_{D}$ are two parameters determined in terms of the parameters of the Hamiltonian by,

$$
\begin{aligned}
\Lambda_{D} & \equiv \sqrt{\frac{G}{2}} \\
& =\frac{1}{2}\left[\sqrt{(D-2)^{2}+2 g}-(D-2)\right] \\
\Gamma_{D} & =\frac{1}{2}\left[D(N-1)-2+\Lambda_{D} N(N-1)\right],
\end{aligned}
$$

where $L_{n}^{\Gamma_{D}}$ denotes the Laguerre polynomial. As expected, the results are in agreement with [1] and [7] for $D=1$ and 3 respectively. It is worth noting here that the case $D=2$ is somewhat special, since the results are valid only for $g \geq 0$. Further, for any $D>1$, as $G$ and $g \longrightarrow 0$, the $n=0$ state corresponds to the ground state of the oscillator problem without the centrifugal barrier and with Bose statistics. Thus the situation is different from the case in one dimension [6], where, as $g \longrightarrow 0$, the ground state is that of the oscillator problem without the centrifugal barrier and with Fermi statistics. It is not clear whether the difference in statistics in the cases of one and more than one dimensions has any deeper physical significance.

Now let us consider a Sutherland variant of the above problem, where now the system is described by the Hamiltonian,

$$
\mathcal{H}=-\sum_{i=1}^{N} \nabla_{i}^{2}+g \sum_{\{i, j \mid i<j\}}^{N} \frac{1}{r_{i j}^{2}}+G \sum_{\substack{\{i, j, k\} \\ i<j, i \neq k, j \neq k}} \frac{\mathbf{r}_{k i} \cdot \mathbf{r}_{k j}}{r_{k i}^{2} r_{k j}^{2}}+\omega^{2} \sum_{i=1}^{N} r_{i}^{2} .
$$

This Hamiltonian is obtained by replacing the $\frac{1}{8} \omega^{2} \sum_{i>j=1}^{N} r_{i j}^{2}$ in (11) with the harmonic well potential $\omega^{2} \sum_{i=1}^{N} r_{i}^{2}$ in the same spirit as in [6].

The ground state eigenfunctions and eigenvalues of the system(7) are given by,

$$
\begin{aligned}
\Psi_{0} & =\hat{C} \exp \left(-\frac{\omega}{2} \sum_{i=1}^{N} r_{i}^{2}\right) \prod_{i<j}\left|\mathbf{r}_{i}-\mathbf{r}_{j}\right|^{\Lambda_{D}}, \\
E_{0} & =\left[D N+N(N-1) \Lambda_{D}\right] \omega,
\end{aligned}
$$

where $\Lambda_{D}$ is as given in (4) and (5). As in the previous case, as $G$ and $g \longrightarrow 0$, the ground state becomes that for non-interacting particles in a harmonic potential, without the centrifugal barrier and with Bose statistics.

Now, rewriting $\Psi_{0}$ from (8) in terms of a new variable

$$
\mathbf{y}_{i} \equiv \sqrt{\frac{\omega}{\Lambda_{D}}} \mathbf{r}_{i}
$$

we have the probability distribution for the $N$ particles as

$$
\Psi_{0}^{2}=C \exp \left(-\Lambda_{D} \sum_{i=1}^{N} y_{i}^{2}\right) \prod_{i<j}\left|\mathbf{y}_{i}-\mathbf{y}_{j}\right|^{2 \Lambda_{D}}
$$


where $C$ is the constant of normalization.

In the case of $D=1$, there is no three-body interaction [7], i.e. $G=0$ and $\Lambda_{1}$ is determined from the definition(5). It was shown in [6] that for $\Lambda_{1}=\frac{1}{2}, 1$ and 2 , the corresponding expressions for $\Psi_{0}^{2}$ match with the distribution functions for the eigenvalues of random matrices from a Gaussian ensemble. In particular, they correspond respectively to the orthogonal, unitary and symplectic matrices [8]. Using the well-known results derived earlier [8], Sutherland [6] wrote down the expressions for density and pair-correlation functions for a many-body system in one dimension.

Our concern for the rest of this letter will be to deal with the case of $D=2$. We shall show that in this case also the expression for $\Psi_{0}^{2}$ renders itself to identification with the distribution of eigenvalues from an ensemble of complex matrices, provided, $g=2$.

In two-dimensions, the ground state energy $(9)$ becomes

$$
E_{0}=\left[2 N+N(N-1) \Lambda_{2}\right] \omega,
$$

while the parameter $\Lambda_{2}$ becomes

$$
\Lambda_{2}=\sqrt{\frac{G}{2}}=\sqrt{\frac{g}{2}}
$$

so that the strengths of both the interactions are equal $(G=g)$. The form of the distribution (11) remains the same, with $\mathbf{y}_{i}$ denoting two-dimensional vectors. Now, one can write two-vectors' as complex numbers, the two-dimensional space of positions of the particles becoming the complex plane, $\mathbb{C}$. Let us denote the complex numbers corresponding to the two-vectors $\mathbf{y}_{i} \in \mathbb{R}^{2}$ by $z_{i} \in \mathbb{C}$, and rewrite (11) in terms of $z_{i}$ as,

$$
\left[\Psi_{0}\left(z_{i}\right)\right]^{2}=C \exp \left(-\Lambda_{2} \sum_{i=1}^{N}\left|z_{i}\right|^{2}\right) \prod_{i<j}\left|z_{i}-z_{j}\right|^{2 \Lambda_{2}},
$$

The form (14) of $\Psi_{0}^{2}$ is known to occur in the study of complex random matrices. This is precisely the form of the joint probability density function of the eigenvalues of matrices from an ensemble of complex matrices [8, 9], provided one sets $\Lambda_{2}$ equal to 1 . The normalization constant can be determined as in 8, 9], and the expression for $\Psi_{0}^{2}$ with $\Lambda_{2}=1$ and normalized to unity is

$$
\left[\Psi_{0}\left(z_{i}\right)\right]^{2}=\left(\pi^{N} \prod_{p=1}^{N} p !\right)^{-1} \exp \left(-\sum_{i=1}^{N}\left|z_{i}\right|^{2}\right) \prod_{i<j}\left|z_{i}-z_{j}\right|^{2} .
$$

It is worth noting here that by fixing $\Lambda_{2}$, one is left with one single dimensional variable in the problem, viz. $\omega$, which has the dimension of $\frac{1}{\text { length }^{2}}$ (recall that we are working in the units $\hbar^{2}=2 m=1$ ). Therefore, the thermodynamic limit of taking the area and $N$ to be large with their ratio kept finite, will be achieved by taking 
$N \longrightarrow \infty, \omega \longrightarrow 0$, with $N \omega=$ constant. We shall show below how to take this singular limit.

Following [8,9] one can also find out the $n$-point correlation functions for all $n$. Let us quote the general result. The $n$-point correlation function is defined as

$$
\mathcal{R}_{n}\left(z_{1}, \cdots, z_{n}\right) \equiv \frac{N !}{(N-n) !} \int \ldots \int\left[\Psi_{0}\left(z_{i}\right)\right]^{2} \prod_{i=n+11}^{N}\left|d z_{i}\right|^{2}
$$

For the case at hand one finds, after calculating the Van-der Monde determinant,that the expression for the $n$-point correlation function is

$$
\mathcal{R}_{n}\left(z_{1}, \cdots, z_{n}\right)=\frac{1}{\pi^{n}} \exp \left(-\sum_{1}^{n}\left|z_{i}\right|^{2}\right) \operatorname{det}\left[\sum_{p=0}^{N-1} \frac{\left(z_{i} z_{j}^{*}\right)^{p}}{p !}\right]_{\{i, j=1,2, \cdots n\}}
$$

As $N \longrightarrow \infty$, the correlation functions tend to well-defined limits:

$$
\mathcal{R}_{n}\left(z_{1}, \cdots, z_{n}\right) \sim \frac{1}{\pi^{n}} \exp \left(-\sum_{1}^{n}\left|z_{i}\right|^{2}\right) \operatorname{det}\left[e^{z_{i} z_{j}^{*}}\right]_{\{i, j=1,2, \cdots n\}} .
$$

In particular, the one-point correlation function, defined as,

$$
\mathcal{R}(z)=N \int_{\mathbb{C}^{N-1}} \Psi_{0}^{2} d z_{2} d z_{3} \cdots d z_{N}
$$

and interpreted as the density of the $N$-particle system, is given by:

$$
\mathcal{R}(\zeta)=\frac{1}{\pi} \exp \left(-\zeta^{2}\right) \sum_{p=0}^{N-1} \frac{\zeta^{2 p}}{p !}
$$

where we have put $|z|=\zeta$ and omitted the suffix 1: $\mathcal{R} \equiv \mathcal{R}_{1}$. The density(20) is isotropic and does not depend on the angular coordinate $\theta \equiv \arg (z)$. The density is normalized as

$$
\int_{0}^{2 \pi} \int_{0}^{\infty} \mathcal{R}(\zeta) \zeta d \zeta d \theta=N
$$

the total number of particles. It is interesting to note the difference between the density (20) and the semi-circular expression for density obtained in [6] for the corresponding one-dimensional system, viz.

$$
\begin{aligned}
\mathcal{R}(y) & =\sqrt{2 N-y^{2}}, & & y^{2}<N, \\
& =0, & & y^{2}>N .
\end{aligned}
$$

Each integral in the sum in $\mathcal{R}(\zeta)$ gives a value of unity and the $N$ terms of the sum adds upto $N$. This behavior is different from the simpler form of density in (22), where one could factor out 
$\sqrt{N}$ from the density. This difference in the form of the density will be manifested in the different way of achieving the thermodynamic limits in the two cases. Let us now write down the expression for the density in terms of the original position vectors r. Demanding the same normalization as in (21), this leads to the following expression for density

$$
\mathcal{R}(r)=\frac{\omega}{\pi \Lambda_{2}} \exp \left(-\omega r^{2}\right) \sum_{p=0}^{N-1} \frac{\left(\omega r^{2}\right)^{p}}{p !}
$$

As noted earlier, the thermodynamic limit of the expressions is given by $N \longrightarrow \infty$ and $\omega \longrightarrow 0$, with $N \omega$ kept constant. However, one can see from the expression(23) that it is not meaningful to take the limit $\omega \longrightarrow 0$, since that will make the density vanishing. In order to take this limit meaningfully, one has to express the density in terms of a new variable, defined by pulling out a factor of $\sqrt{N}$ from $\mathbf{r}$,

$$
\mathbf{r}=\sqrt{N} \boldsymbol{\rho}
$$

The density $\mathcal{R}(\rho)$ with the same normalization takes the form:

$$
\mathcal{R}(\rho)=\frac{\omega N}{\pi} \exp \left(-N \omega \rho^{2}\right) \sum_{p=0}^{N-1} \frac{\left(N \omega \rho^{2}\right)^{p}}{p !} .
$$

Denoting the density at $\rho=0$ by $\mathcal{R}_{0}$, we find that $\mathcal{R}_{0}=\frac{\omega N}{\pi}$. Thus, we see that for a fixed $\mathcal{R}_{0}$, letting $N \longrightarrow \infty$ means $\omega \longrightarrow 0$ as $\frac{1}{N}$.

The pair-correlation function for the system has also been obtained [8, 9]. It is given by

$$
\mathcal{R}_{2}(\xi)=\mathcal{R}_{0}^{2}\left[1-\exp \left(-\pi \xi^{2}\right)\right]
$$

where $\xi=\frac{\sqrt{N \omega}}{\pi} \rho_{i j}$. Let us note at this point that, the pair-correlation function, as given above in (26), has apparent similarity with the pair-correlation function for the corresponding one dimensional case [6], with $\Lambda_{1}=\frac{1}{2}$, which corresponds to the case of orthogonal Gaussian ensemble. In both the cases the function $\mathcal{R}$ starts off from a value of 0 near $\xi=0$ and grows up to unity as $\xi \longrightarrow \infty$. To facilitate discussion let us compare the functions $Y_{2}=1-\mathcal{R}_{2}$ setting $\mathcal{R}_{0}=1$ in both the cases. The behavior of $Y_{2}$ for our case is

$$
Y_{2}(\xi)=1-\pi \xi^{2}+\frac{\pi^{2} \xi^{4}}{2}-\frac{\pi^{3} \xi^{6}}{6}+\cdots
$$

while for the orthogonal $\left(\Lambda_{1}=\frac{1}{2}\right)$ case of [6], one had

$$
Y_{2}(\xi)=1-\frac{\pi^{2} \xi}{6}+\frac{\pi^{3} \xi^{3}}{60}-\frac{\pi^{4} \xi^{4}}{135}+\cdots
$$


That is, while for small $\xi, Y_{2}$ goes in a power series with only even power of $\xi$ in our case, for the $\Lambda_{1}=\frac{1}{2}$ case in one dimension, it is a power series in all powers of $\xi$. However, for large distances, while the $Y_{2}$ in [ [6] goes as powers of $\frac{1}{\xi^{2}}$, i.e.

$$
Y_{2}(\xi)=\frac{1}{\pi^{2} \xi^{2}}-\frac{1+\cos ^{2} \pi \xi}{\pi^{4} \xi^{4}}+\cdots,
$$

with extremely mild oscillations, we have a Gaussian fall-off: $Y_{2}=\exp \left(-\xi^{2}\right)$,

without any oscillations. Moreover, since the Fourier transform of a Gaussian function is a Gaussian only, the form factor for our system is a Gaussian, unlike that in $[6]$.

One can also write down the higher order correlations [10]. For example, the three-particle correlation function in the variables $\boldsymbol{\rho}$ is given by:

$$
\begin{aligned}
& \mathcal{R}_{3}\left(\boldsymbol{\rho}_{\mathbf{1}}, \boldsymbol{\rho}_{\mathbf{2}}, \boldsymbol{\rho}_{\mathbf{3}}\right) / \mathcal{R}_{0}^{3}=1-\exp \left(-\pi \mathcal{R}_{0} \rho_{12}^{2}\right)-\exp \left(-\pi \mathcal{R}_{0} \rho_{23}^{2}\right)-\exp \left(-\pi \mathcal{R}_{0} \rho_{31}^{2}\right) \\
+ & 2 \exp \left[-\frac{1}{2} \pi \mathcal{R}_{0}\left(\rho_{12}^{2}+\rho_{23}^{2}+\rho_{31}^{2}\right)\right] \cos \left[2 \pi \mathcal{R}_{0} \mathcal{A}(1,2,3)\right],
\end{aligned}
$$

where $\mathcal{A}(1,2,3)$ is the area of the triangle formed by the three particles 1,2 and 3 . The four-body correlation function is

$$
\begin{aligned}
& \mathcal{R}_{4}\left(\boldsymbol{\rho}_{\mathbf{1}}, \boldsymbol{\rho}_{\mathbf{2}}, \boldsymbol{\rho}_{\mathbf{3}} \boldsymbol{\rho}_{4}\right) / \mathcal{R}_{0}^{4}=1-\exp \left(-\pi \mathcal{R}_{0} \rho_{12}^{2}\right) \cdots \\
+ & \exp \left[-\pi \mathcal{R}_{0}\left(\rho_{12}^{2}+\rho_{34}^{2}\right)\right]+\cdots \\
+ & 2 \exp \left[-\frac{1}{2} \pi \mathcal{R}_{0}\left(\rho_{12}^{2}+\rho_{23}^{2}+\rho_{31}^{2}\right)\right] \cos \left[2 \pi \mathcal{R}_{0} \mathcal{A}(1,2,3)\right]+\cdots \\
- & 2 \exp \left[-\frac{1}{2} \pi \mathcal{R}_{0}\left(\rho_{12}^{2}+\rho_{23}^{2}+\rho_{34}^{2}+\rho_{41}^{2}\right)\right] \cos \left[2 \pi \mathcal{R}_{0} \mathcal{A}(1,2,3,4)\right],
\end{aligned}
$$

where $\mathcal{A}(1,2,3,4) \equiv \frac{1}{2}\left|\boldsymbol{\rho}_{\mathbf{1 3}} \times \boldsymbol{\rho}_{\mathbf{2 4}}\right|$ and $\cdots$ stands for permutations of the indices. It is obvious from (30) and (31 ) as well as from the general expression(17) for the $n$-point correlation functions that all the distribution functions are isotropic, i.e., do not depend on the angular coordinate. Further, they do not show either long-range or quasi-long-range order and have a Gaussian fall-off at large distances.

The form of the distribution function (11) is the same [10] as that occurs in the case of two-dimensional Coulomb gas [11, 12], although the physics in the two cases are quite different.

To conclude, in this letter, we have studied a many-body problem in two dimensions. We have written down explicit expressions for the ground state energy and the pair-correlation function, in case the strength of the inverse square interaction is set to $g=2$. We then show that in this case, there is no long-range or quasi-long-range order. It would be very interesting if one could obtain exact results for other values of $g$ and see if there is long-range order for any value of $g$. It will also be interesting if one could solve a similar many-body problem in higher dimensions, especially in three dimensions. But to our knowledge, the corresponding random matrix problem has not been worked out as yet. 


\section{Note Added:}

After this letter was accepted for publication, we became aware of the work of Girvin and MacDonald [13], where they showed that the gauge-transformed Laughlin wavefunction [ eq. (7) of their paper] shows off-diagonal long-range order. It then immediately follows that the Calogero-Sutherland ground state wave-function in two dimensions as given by (8) [which is identical to eq. (7) of [13]] also exhibits offdiagonal long-range order.

\section{References}

[1] F Calogero, J. Math Phys. 10(1969)2191,2197; ibid. 12(1971)419.

[2] M Olshanetsky and A Perelomov, Phys. Rep.71(1981)313; ibid. 94(1983)313.

[3] B Simons, P Lee and B Altshuler, Phys. Rev. Lett.72(1994)64 .

[4] A Khare, J. Phys. A 29(1996)L45.

[5] P Ghosh, To appear.

[6] B Sutherland, J. Math Phys. 12(1971)246.

[7] F Calogero and C Marchioro, J. Math Phys. 14(1973)182.

[8] M L Mehta, Random Matrices, Second Edition, Academic Press, NY(1991).

[9] J Ginibre, J. Math Phys. 6(1965)440.

[10] B Jancovici, Phys. Rev. Lett.46(1981)386.

[11] F Dyson, J. Math Phys. 3(1962)140,157,166.

[12] E Wigner, Proc 4th Can Math Cong, Toronto, 1959; p 174.

[13] S Girvin and A McDonald, Phys. Rev. Lett.58(1987)1252. 